\title{
The Relaxed Gradient Based Iterative Algorithm for the Symmetric (Skew Symmetric) Solution of the Sylvester Equation $A X+X B=C$
}

\author{
Xiaodan Zhang ${ }^{1}$ and Xingping Sheng ${ }^{2}$ \\ ${ }^{1}$ School of Information and Computer, Anhui Agricultural University, Hefei 230036, China \\ ${ }^{2}$ School of Mathematics and Statistics, Fuyang Normal College, Anhui 236037, China \\ Correspondence should be addressed to Xingping Sheng; xingpingsheng@163.com
}

Received 26 February 2017; Accepted 23 March 2017; Published 3 April 2017

Academic Editor: Jean Jacques Loiseau

Copyright ( 2017 Xiaodan Zhang and Xingping Sheng. This is an open access article distributed under the Creative Commons Attribution License, which permits unrestricted use, distribution, and reproduction in any medium, provided the original work is properly cited.

\begin{abstract}
In this paper, we present two different relaxed gradient based iterative (RGI) algorithms for solving the symmetric and skew symmetric solution of the Sylvester matrix equation $A X+X B=C$. By using these two iterative methods, it is proved that the iterative solution converges to its true symmetric (skew symmetric) solution under some appropriate assumptions when any initial symmetric (skew symmetric) matrix $X_{0}$ is taken. Finally, two numerical examples are given to illustrate that the introduced iterative algorithms are more efficient.
\end{abstract}

\section{Introduction}

For the convenience of our statements, the following notations will be used throughout the paper: $R^{m \times n}$ represents the set of $m \times n$ real matrices. For $A \in R^{m \times n}$, we write $A^{T}, R(A), \operatorname{tr}(A), \rho(A), \lambda_{\max }(A), \lambda_{\min }(A),\|A\|_{2}$, and $\|A\|_{F}$ to denote the transpose, the range space, the trace, the spectral radius, the maximal eigenvalue, the minimal eigenvalue, the spectral norm, and the Frobenius norm of a matrix $A$, respectively; that is, $\|A\|_{2}=\sqrt{\lambda_{\max }\left(A^{T} A\right)}$ and $\|A\|_{F}=$ $\left[\operatorname{tr}\left(A^{T} A\right)\right]^{1 / 2} \cdot \sigma_{\max }(A), \sigma_{\min }(A)$ are the maximal singular value and the minimal nonzero singular value of $A$. The symbol of $I_{n}$ represents an identity matrix of order $n, \mathbf{1}_{m \times n}$ is an $m \times n$ matrix whose elements are 1 , and $\operatorname{cond}(A)=$ $\sigma_{\max }(A) / \sigma_{\min }(A)$ is the condition number of matrix $A$. The inner product in space $R^{m \times n}$ is defined as $\langle A, B\rangle=\operatorname{tr}\left(A^{T} B\right)$; particularly, $\|A\|_{F}^{2}=\operatorname{tr}\left(A^{T} A\right)=\operatorname{tr}\left(A A^{T}\right)=\langle A, A\rangle$. $A \otimes B$ denotes the Kronecker product, defined as $A \otimes B=$ $\left(a_{i j} B\right), i=1, \ldots, m, j=1, \ldots, n$. For any matrix $X=$ $\left(x_{1}, x_{2}, \ldots, x_{n}\right) \in R^{m \times n}$, the vector operator is defined as $\operatorname{vec}(X)=\left(x_{1}^{T}, x_{2}^{T}, \ldots, x_{n}^{T}\right)^{T} \in R^{m n}$. Using vector operator and Kronecker product, we have vec $(A X B)=\left(B^{T} \otimes A\right) \operatorname{vec}(X)$.
Considering the symmetric (skew symmetric) solution of the Sylvester matrix equation

$$
A X+X B=C,
$$

where $A \in R^{n \times n}, B \in R^{n \times n}, C \in R^{n \times n}$, and the unknown matrix $X \in R^{n \times n}$.

The Sylvester matrix equation (1) has many applications in linear system theory, for example, pole/eigenstructure assignment [1-4], robust pole assignment [5-8], robust partial pole assignment [9], observer design [10], model matching problem [11], regularization of descriptor systems [12, 13], disturbance decoupling problem [14], and noninteracting control [15].

As is well known, (1) has a unique solution if and only if $A$ and $-B$ possess no common eigenvalues [16] and the solution can be computed by solving a linear system $(I \otimes$ $\left.A+B^{T} \otimes I\right) \operatorname{vec}(X)=\operatorname{vec}(C)$. Using this method, it will increase the computational cost and storage requirements, so that this approach is only applicable for small sized Sylvester equations. 
Due to these drawbacks, many other methods for the solution have appeared in the literature. The idea of transforming the coefficient matrix into a Schur or Hessenberg form to compute (1) have been presented in $[16,17]$. When the linear matrix (1) is inconsistent, a finite iterative method to solving its Hermitian minimum norm solutions has been presented in [18]. An efficient iterative method based on Hermitian and skew Hermitian splitting has been proposed in [19]. Krylov subspace based methods have been presented in [20-26] for solving Sylvester equations and generalized Sylvester equations. Recently based on the idea of a hierarchical identification principle [27-29], some efficient gradient based iterative algorithms for solving generalized Sylvester equations and coupled (general coupled) Sylvester equations have been proposed in [27, 30-32]. Particularly, for Sylvester equations of form (1), it is illustrated in [33] that the unknown matrix to be identified can be computed by a gradient based iterative algorithm. The convergence properties of the methods are investigated in $[27,32]$. Niu et al. [34] proposed a relaxed gradient based iterative algorithm for solving Sylvester equations. Wang et al. [35] proposed a modified gradient based iterative algorithm for solving Sylvester equations (1). More recently, Xie and Ma [36] gave an accelerated gradient based iterative algorithm for solving (1). In [37, 38] Xie et al. studied the special structure solution of matrix equation (1) by using iterative method.

In this paper, inspired by [28, 34-36], we first derive a relaxed gradient based iterative (RGI) algorithm for solving the symmetric solution of matrix equation (1). Theoretical analysis shows that our method converges to the exact symmetric solution for any initial value with some appropriate assumptions. Then the proposed algorithm can be also applied to the skew symmetric solution of matrix equation (1). Numerical results illustrate that the proposed method is correct and feasible. We must point out that the ideas in this paper have some differences comparing with that in $[28,34-$ 36].

The rest of the paper is organized as follows. In Section 2, some main preliminaries are provided. In Section 3, the relaxed gradient based iterative methods are studied. Finally, a numerical example is included to verify the superior convergence for the algorithms.

\section{Preliminaries}

In this section, we reviewed the ideas and principles of the gradient based iterative (GI) method, the relaxed gradient based iterative (RGI) method, and the modified gradient based iterative (MGI) method.

Let $\mu>0$ be the convergence factor or step factor. The gradient based iterative method for $A x=b$ is as follows:

$$
x(k)=x(k-1)-\mu A^{T}(A x(k-1)-b) .
$$

The convergence of the gradient based iterative is stated as follows.

Lemma 1 (see [32]). Assume matrix $A$ is full column-rank and $0<\mu<2 /\|A\|_{2}^{2}$; then the gradient based iterative sequences $\{x(k)\}$ in (2) converge to $x^{*}$; that is, $\lim _{k \rightarrow \infty} x(k)=x^{*}$ or the error $\tilde{x}(k)=x(k)-x^{*}$ converges to zero for any initial value $x(0)$. Moreover, the maximal 2 -convergence rate is given by $\gamma_{\max }=1 / \beta\left(\mu_{\text {opt }}\right)$, where

$$
\begin{aligned}
\beta\left(\mu_{o p t}\right) & =\frac{\operatorname{cond}^{2}(A)-1}{\operatorname{cond}^{2}(A)+1}, \\
\mu_{o p t} & =\frac{2}{\sigma_{\text {max }}^{2}(A)+\sigma_{\text {min }}^{2}(A)} .
\end{aligned}
$$

In this case, the error vector $\tilde{x}(k)$ satisfies

$$
\|\tilde{x}(k)\| \leq \beta^{k}\left(\mu_{o p t}\right)\|\tilde{x}(0)\|, \quad k \geq 0 .
$$

In [28], Ding and Chen presented the following algorithm based on gradient for solving (1).

Algorithm 2 (see [28] (the gradient based iterative (GI) algorithm)).

Step 1. Input matrices $A, B, C \in R^{n \times n}$, given any small positive number $\epsilon$. Choose the initial matrices $X_{1}(0)$ and $X_{2}(0)$. Compute $X(0)=\left(X_{1}(0)+X_{2}(0)\right) / 2$. Set $k:=1$.

Step 2. If $\delta_{k}=\|A X(k)+X(k) B-C\|_{F} /\|C\|_{F}<\epsilon$, stop; otherwise, go to Step 3.

Step 3. Update the sequences

$$
\begin{aligned}
X_{1}(k)= & X(k-1) \\
& -\mu A^{T}[A X(k-1)+X(k-1) B-C], \\
X_{2}(k)= & X(k-1) \\
& -\mu[A X(k-1)+X(k-1) B-C] B^{T}, \\
X(k)= & \frac{X_{1}(k)+X_{2}(k)}{2} .
\end{aligned}
$$

Step 4. Set $k:=k+1$; return to Step 2 .

The authors [28] also pointed out that if the convergence factor $\mu$ is chosen in $\left(0,2 /\left(\sigma^{2}(A)+\sigma^{2}(B)\right)\right)$, Algorithm 2 will converge to the exact solution of (1).

Niu et al. [34] gave a relaxed gradient based iterative algorithm for solving (1). When $\mu$ is in $\left(0,2 / \omega(1-\omega)\left(\sigma^{2}(A)+\right.\right.$ $\left.\left.\sigma^{2}(B)+\sigma\left(B A^{T}\right)\right)\right)$ the following algorithm has been proven to be convergent.

Algorithm 3 (see [34] (the relaxed gradient based iterative (RGI) algorithm)).

Step 1. Input matrices $A, B, C \in R^{n \times n}$, given any small positive number $\epsilon$ and appropriate positive number $\omega$. Choose the initial matrices $X_{1}(0)$ and $X_{2}(0)$. Compute $X(0)=\omega X_{1}(0)+$ $(1-\omega) X_{2}(0)$. Set $k:=1$.

Step 2. If $\delta_{k}=\|A X(k)+X(k) B-C\|_{F} /\|C\|_{F}<\epsilon$, stop; otherwise, go to Step 3. 
Step 3. Update the sequences

$$
\begin{aligned}
& X_{1}(k) \\
& =X(k-1) \\
& -(1-\omega) \mu A^{T}[A X(k-1)+X(k-1) B-C], \\
& X_{2}(k) \\
& =X(k-1) \\
& -\omega \mu[A X(k-1)+X(k-1) B-C] B^{T}, \\
& X(k)=\omega X_{1}(k)+(1-\omega) X_{2}(k) \text {. }
\end{aligned}
$$

Step 4. Set $k:=k+1$; return to Step 2 .

Recently, in [35] Wang et al. proposed a modified gradient based iterative (MGI) algorithm to solve (1). The main difference is that, in the step of computing $X_{2}(k)$, the last approximate solution $X_{1}(k)$ has been considered fully to update $X(k-1)$.

Algorithm 4 (see [35] (the modified gradient based iterative (MGI) algorithm)).

Step 1. Input matrix $A, B, C \in R^{n \times n}$, given any small positive number $\epsilon$ and appropriate positive number $\omega$. Choose the initial matrices $X_{1}(0)$ and $X_{2}(0)$. Compute $X(0)=\left(X_{1}(0)+\right.$ $\left.X_{2}(0)\right) / 2$. Set $k:=1$.

Step 2. If $\delta_{k}=\|A X(k)+X(k) B-C\|_{F} /\|C\|_{F}<\epsilon$, stop; otherwise, go to Step 3.

Step 3. Update the sequences

$$
\begin{aligned}
X_{1}(k)= & X(k-1) \\
& -\mu A^{T}[A X(k-1)+X(k-1) B-C] .
\end{aligned}
$$

Step 4. Compute

$$
X(k-1)=\frac{X_{1}(k)+X_{2}(k-1)}{2} .
$$

Step 5. Update the sequences

$$
\begin{aligned}
X_{2}(k)= & X(k-1) \\
& -\mu[A X(k-1)+X(k-1) B-C] B^{T} .
\end{aligned}
$$

Step 6. Compute

$$
X(k)=\frac{X_{1}(k)+X_{2}(k)}{2} .
$$

Step 7. Set $k:=k+1$; return to Step 2 .
More recently, Xie and Ma [36] presented the following AGBI algorithm for solving (1) based on the idea of MGI.

Algorithm 5 (see [36] (the accelerated gradient based iterative (AGBI) algorithm)).

Step 1. Input matrix $A, B, C \in R^{n \times n}$, given any small positive number $\epsilon$ and appropriate positive number $\omega$. Choose the initial matrices $X_{1}(0)$ and $X_{2}(0)$. Compute $X(0)=\omega X_{1}(0)+$ $(1-\omega) X_{2}(0)$. Set $k:=1$.

Step 2. If $\delta_{k}=\|A X(k)+X(k) B-C\|_{F} /\|C\|_{F}<\epsilon$, stop; otherwise, go to Step 3.

Step 3. Update the sequences

$$
\begin{aligned}
& X_{1}(k) \\
& =X(k-1) \\
& \quad-(1-\omega) \mu A^{T}[A X(k-1)+X(k-1) B-C] .
\end{aligned}
$$

Step 4. Compute

$$
X(k-1)=\omega X_{1}(k)+(1-\omega) X_{2}(k-1) .
$$

Step 5. Update the sequences

$$
\begin{aligned}
X_{2}(k)= & X(k-1) \\
& -\omega \mu[A X(k-1)+X(k-1) B-C] B^{T} .
\end{aligned}
$$

Step 6. Compute

$$
X(k)=\omega X_{1}(k)+(1-\omega) X_{2}(k) .
$$

Step 7. Set $k:=k+1$; return to Step 2 .

\section{Main Results}

In this section, we first study the necessary and sufficient conditions of the symmetric solution for (1). Then the relaxed gradient based iterative algorithm for the symmetric solution of equation (1) is proposed. Following the same line, the relaxed gradient based iterative algorithm for the skew symmetric solution of equation (1) is also presented.

Theorem 6. The matrix equation (1) has a unique symmetric solution $X^{s}$ if and only if the following pair of the matrix equations

$$
\begin{aligned}
A X+X B & =C, \\
B^{T} X+X A^{T} & =C^{T}
\end{aligned}
$$

has a unique common solution $X^{*}$ and $X^{s}=\left(X^{*}+\left(X^{*}\right)^{T}\right) / 2$. 
Proof. If $X^{s}$ is a unique symmetric solution of (1), then $\left(X^{s}\right)^{T}=X^{s}$ and $A X^{s}+X^{s} B=C$; further we have

$$
B^{T} X^{s}+X^{s} A^{T}=\left(A X^{s}+X^{s} B\right)^{T}=C^{T} .
$$

This shows that $X^{s}$ is also the solution of the pair matrix equations (15).

Conversely, if the system of matrix equations (15) has a common solution $X^{*}$, let us denote $X^{s}=\left(X^{*}+\left(X^{*}\right)^{T}\right) / 2$; then we can check that

$$
\begin{aligned}
A X^{s}+X^{s} B & =A \frac{X^{*}+\left(X^{*}\right)^{T}}{2}+\frac{X^{*}+\left(X^{*}\right)^{T}}{2} B \\
& =\frac{A X^{*}+X^{*} B}{2}+\frac{A\left(X^{*}\right)^{T}+\left(X^{*}\right)^{T} B}{2} \\
& =\frac{A X^{*}+X^{*} B}{2}+\frac{\left(B^{T} X^{*}+X^{*} A^{T}\right)^{T}}{2} \\
& =\frac{C+\left(C^{T}\right)^{T}}{2}=C .
\end{aligned}
$$
(1).

This implies that $X^{s}$ is the unique symmetric solution of

According to Theorem 6, if the unique common solution $X^{*}$ of equations (15) can be obtained, then the unique symmetric solution of $(1)$ is $X^{s}=\left(X^{*}+\left(X^{*}\right)^{T}\right) / 2$.

According to Theorem 6, we construct a relaxed gradient based iterative algorithm to solve the symmetric solution of (1).

Algorithm 7 (the relaxed gradient based iterative (RGI) algorithm for symmetric solution of (1)).

Step 1. Input matrices $A, B, C \in R^{n \times n}$, given any small positive number $\epsilon$ and appropriative positive number $\omega$ such that $0<$ $\omega<1$. Choose any initial matrix $X(0)$. Set $k:=1$.

Step 2. If $\delta_{k}=\left\|A X^{s}(k)+X^{s}(k) B-C\right\|_{F} /\|C\|_{F}<\epsilon$, stop; otherwise, go to Step 3.

Step 3. Update the sequences

$$
\begin{aligned}
& X_{1}(k)=X(k-1) \\
& -\mu_{1}\left[A^{T}(A X(k-1)+X(k-1) B-C)\right. \\
& \left.+(A X(k-1)+X(k-1) B-C) B^{T}\right], \\
& X_{2}(k)=X(k-1) \\
& -\mu_{2}\left[B\left(B^{T} X(k-1)+X(k-1) A^{T}-C^{T}\right)\right. \\
& \left.+\left(B^{T} X(k-1)+X(k-1) A^{T}-C^{T}\right) A\right], \\
& X(k)=\omega X_{1}(k)+(1-\omega) X_{2}(k), \\
& X^{s}(k)=\frac{X(k)+X^{T}(k)}{2} .
\end{aligned}
$$

Step 4. Set $k:=k+1$; return to Step 2 .

In the following paragraph, we will investigate the convergence of Algorithm 7.

Theorem 8. Assume the matrix equations (15) have a unique solution $X^{*}$; then the iterative sequence $\{X(k)\}$ generated by the Algorithm 7 converges to $X^{*}$, if

$$
\begin{aligned}
& 0<\mu_{1}<\frac{1}{\omega\left(\|A\|_{2}^{2}+\|B\|_{2}^{2}\right)} ; \\
& 0<\mu_{2}<\frac{1}{(1-\omega)\left(\|A\|_{2}^{2}+\|B\|_{2}^{2}\right)}
\end{aligned}
$$

that is, $\lim _{k \rightarrow \infty} X(k)=X^{*}$ or the error $X(k)-X^{*}$ converges to zero for any initial value $X(0)$.

Further the sequence $\left\{X^{s}(k)\right\}$ converges to $X^{s}$, where $X^{s}$ is the unique symmetric solution of (1).

Proof. Define the error matrix

$$
\begin{aligned}
\widetilde{X}(k) & =X(k)-X^{*}, \\
\theta_{1}(k-1) & :=A \widetilde{X}(k-1)+\widetilde{X}(k-1) B, \\
\theta_{2}(k-1) & :=B^{T} \widetilde{X}(k-1)+\widetilde{X}(k-1) A^{T} .
\end{aligned}
$$

We have

$$
\begin{aligned}
\widetilde{X}(k) & =X(k)-X^{*}=\omega X_{1}(k)+(1-\omega) X_{2}(k)-X^{*} \\
= & \omega\left(X_{1}(k)-X^{*}\right)+(1-\omega)\left(X_{2}(k)-X^{*}\right) \\
& =\omega\{X(k-1) \\
& -\mu_{1}\left[A^{T}(A X(k-1)+X(k-1) B-C)\right. \\
+ & \left.\left.(A X(k-1)+X(k-1) B-C) B^{T}\right]-X^{*}\right\}+(1 \\
& -\omega)\{X(k-1) \\
& -\mu_{2}\left[B\left(B^{T} X(k-1)+X(k-1) A^{T}-C^{T}\right)\right. \\
+ & \left.\left.\left(B^{T} X(k-1)+X(k-1) A^{T}-C^{T}\right) A\right]-X^{*}\right\} \\
& =\widetilde{X}(k-1)-\left[\omega \mu_{1} A^{T}(A \widetilde{X}(k-1)+\widetilde{X}(k-1) B)\right. \\
+ & (A \widetilde{X}(k-1)+\widetilde{X}(k-1) B) B^{T}+(1-\omega) \\
& +\mu_{2} B\left(B^{T} \widetilde{X}(k-1)+\widetilde{X}(k-1) A^{T}\right) \\
+ & \left.\left(B^{T} \widetilde{X}(k-1)+\widetilde{X}(k-1) A^{T}\right) A\right]=\widetilde{X}(k-1) \\
& -\omega \mu_{1}\left[A^{T} \theta_{1}(k-1)+\theta_{1}(k-1) B^{T}\right]-(1-\omega) \\
& \mu_{2}\left[B \theta_{2}(k-1)+\theta_{2}(k-1) A\right] .
\end{aligned}
$$


The following two equalities are easily derived.

$$
\begin{aligned}
& \left\|A^{T} \theta_{1}(k-1)+\theta_{1}(k-1) B^{T}\right\|_{F}^{2} \\
& \leq\left(\|A\|_{2}^{2}+\|B\|_{2}^{2}\right)\left\|\theta_{1}(k-1)\right\|_{F}^{2}, \\
& \left\|B \theta_{2}(k-1)+\theta_{2}(k-1) A\right\|_{F}^{2} \\
& \leq\left(\|A\|_{2}^{2}+\|B\|_{2}^{2}\right)\left\|\theta_{2}(k-1)\right\|_{F}^{2} .
\end{aligned}
$$

\section{Moreover}

$$
\begin{aligned}
& \|\widetilde{X}(k)\|_{F}^{2}=\| \widetilde{X}(k-1) \\
& -\omega \mu_{1}\left[A^{T} \theta_{1}(k-1)+\theta_{1}(k-1) B^{T}\right]-(1-\omega) \\
& \cdot \mu_{2}\left[B \theta_{2}(k-1)+\theta_{2}(k-1) A\right]\left\|_{F}^{2}=\right\| \widetilde{X}(k-1) \|_{F}^{2} \\
& +\omega^{2} \mu_{1}^{2}\left\|A^{T} \theta_{1}(k-1)+\theta_{1}(k-1) B^{T}\right\|_{F}^{2}+(1-\omega)^{2} \\
& \cdot \mu_{2}^{2}\left\|B \theta_{2}(k-1)+\theta_{2}(k-1) A\right\|_{F}^{2} \\
& -2 \omega \mu_{1} \operatorname{tr}\left[\widetilde{X}(k-1)^{T}\right. \\
& \left.\cdot\left(A^{T} \theta_{1}(k-1)+\theta_{1}(k-1) B^{T}\right)\right]-2(1-\omega) \\
& \cdot \mu_{2} \operatorname{tr}\left[\widetilde{X}(k-1)^{T}\left(B \theta_{2}(k-1)+\theta_{2}(k-1) A\right)\right] \\
& +2 \omega(1-\omega) \\
& \cdot \mu_{1} \mu_{2} \operatorname{tr}\left[\left(A^{T} \theta_{1}(k-1)+\theta_{1}(k-1) B^{T}\right)^{T}\right. \\
& \left.\cdot\left(B \theta_{2}(k-1)+\theta_{2}(k-1) A\right)\right] \leq\|\widetilde{X}(k-1)\|_{F}^{2} \\
& +\omega^{2} \mu_{1}^{2}\left(\|A\|_{2}^{2}+\|B\|_{2}^{2}\right)\left\|\theta_{1}(k-1)\right\|_{F}^{2}+(1-\omega)^{2} \\
& \cdot \mu_{2}^{2}\left(\|A\|_{2}^{2}+\|B\|_{2}^{2}\right)\left\|\theta_{2}(k-1)\right\|_{F}^{2} \\
& -2 \omega \mu_{1}\left\|\theta_{1}(k-1)\right\|_{F}^{2}-2(1-\omega) \mu_{2}\left\|\theta_{2}(k-1)\right\|_{F}^{2} \\
& +2 \omega(1-\omega) \mu_{1} \mu_{2}\left\langle A^{T} \theta_{1}(k-1)+\theta_{1}(k-1)\right. \\
& \left.\cdot B^{T}, B \theta_{2}(k-1)+\theta_{2}(k-1) A\right\rangle \leq\|\widetilde{X}(k-1)\|_{F}^{2} \\
& +\omega^{2} \mu_{1}^{2}\left(\|A\|_{2}^{2}+\|B\|_{2}^{2}\right)\left\|\theta_{1}(k-1)\right\|_{F}^{2}+(1-\omega)^{2} \\
& \cdot \mu_{2}^{2}\left(\|A\|_{2}^{2}+\|B\|_{2}^{2}\right)\left\|\theta_{2}(k-1)\right\|_{F}^{2} \\
& -2 \omega \mu_{1}\left\|\theta_{1}(k-1)\right\|_{F}^{2}-2(1-\omega) \mu_{2}\left\|\theta_{2}(k-1)\right\|_{F}^{2} \\
& +2 \omega \mu_{1}\left\|A^{T} \theta_{1}(k-1)+\theta_{1}(k-1) B^{T}\right\|_{F}(1-\omega) \\
& \cdot \mu_{2}\left\|B \theta_{2}(k-1)+\theta_{2}(k-1) A\right\|_{F} \leq\|\widetilde{X}(k-1)\|_{F}^{2} \\
& +\omega^{2} \mu_{1}^{2}\left(\|A\|_{2}^{2}+\|B\|_{2}^{2}\right)\left\|\theta_{1}(k-1)\right\|_{F}^{2}+(1-\omega)^{2} \\
& \cdot \mu_{2}^{2}\left(\|A\|_{2}^{2}+\|B\|_{2}^{2}\right)\left\|\theta_{2}(k-1)\right\|_{F}^{2}
\end{aligned}
$$

$$
\begin{aligned}
& -2 \omega \mu_{1}\left\|\theta_{1}(k-1)\right\|_{F}^{2}-2(1-\omega) \mu_{2}\left\|\theta_{2}(k-1)\right\|_{F}^{2} \\
& +\omega^{2} \mu_{1}^{2}\left\|A^{T} \theta_{1}(k-1)+\theta_{1}(k-1) B^{T}\right\|_{F}^{2}+(1-\omega)^{2} \\
& \cdot \mu_{2}^{2}\left\|B \theta_{2}(k-1)+\theta_{2}(k-1) A\right\|_{F}^{2}=\|\widetilde{X}(k-1)\|_{F}^{2} \\
& -2 \omega \mu_{1}\left[1-\omega \mu_{1}\left(\|A\|_{2}^{2}+\|B\|_{2}^{2}\right)\right]\left\|\theta_{1}(k-1)\right\|_{F}^{2} \\
& -2(1-\omega) \mu_{2}\left[1-(1-\omega) \mu_{2}\left(\|A\|_{2}^{2}+\|B\|_{2}^{2}\right)\right]
\end{aligned}
$$$$
\cdot\left\|\theta_{2}(k-1)\right\|_{F}^{2} \text {. }
$$

Therefore, the above equality implies that

$$
\begin{aligned}
\|\widetilde{X}(k)\|_{F}^{2} \leq\|\widetilde{X}(0)\|_{F}^{2}-2 \omega \mu_{1}\left[1-\omega \mu_{1}\left(\|A\|_{2}^{2}+\|B\|_{2}^{2}\right)\right] \\
\cdot \sum_{i=1}^{k}\left\|\theta_{1}(i-1)\right\|_{F}^{2}-2(1-\omega) \\
\cdot \mu_{2}\left[1-(1-\omega) \mu_{2}\left(\|A\|_{2}^{2}+\|B\|_{2}^{2}\right)\right] \\
\cdot \sum_{i=1}^{k}\left\|\theta_{2}(i-1)\right\|_{F}^{2} .
\end{aligned}
$$

It follows from $0<\mu_{1}<1 / \omega\left(\|A\|_{2}^{2}+\|B\|_{2}^{2}\right)$ and $0<\mu_{2}<$ $1 /(1-\omega)\left(\|A\|_{2}^{2}+\|B\|_{2}^{2}\right)$ that

$$
\begin{aligned}
& \sum_{i=1}^{\infty}\left\|\theta_{1}(i-1)\right\|_{F}^{2}<\infty, \\
& \sum_{i=1}^{\infty}\left\|\theta_{2}(i-1)\right\|_{F}^{2}<\infty .
\end{aligned}
$$

The above two inequalities (25) mean that

$$
\begin{aligned}
& \lim _{k \rightarrow \infty} \theta_{1}(k-1)=0, \\
& \lim _{k \rightarrow \infty} \theta_{2}(k-1)=0 .
\end{aligned}
$$

In other words

$$
\begin{aligned}
\lim _{k \rightarrow \infty}[A X(k-1)+X(k-1) B] & =C, \\
\lim _{k \rightarrow \infty}\left[B^{T} X(k-1)+X(k-1) A^{T}\right] & =C^{T} .
\end{aligned}
$$

Equation (27) implies that

$$
\lim _{k \rightarrow \infty} X(k)=X^{*} .
$$

From Theorem 6 and the above limited equation (28), we have

$$
\begin{aligned}
\lim _{k \rightarrow \infty} X^{s}(k) & =\lim _{k \rightarrow \infty} \frac{X(k)+X^{T}(k)}{2}=\frac{X^{*}+\left(X^{*}\right)^{T}}{2} \\
& =X^{s}
\end{aligned}
$$


Following the same line, the idea of Algorithm 7 can be extended to solve the skew symmetric solution of (1). First, we need the following theorem.

Theorem 9. The matrix equation (1) has a unique skew symmetric solution $X^{s s}$ if and only if the following pair of the matrix equations

$$
\begin{gathered}
A X+X B=C, \\
B^{T} X+X A^{T}=-C^{T}
\end{gathered}
$$

has a unique common solution $X^{*}$ and $X^{s s}=\left(X^{*}-\left(X^{*}\right)^{T}\right) / 2$.

The relaxed gradient based iterative algorithm for solving the skew symmetric solution of (1) can be stated as follows.

Algorithm 10 (the relaxed gradient based iterative (RGI) algorithm for skew symmetric solution of (1)).

Step 1. Input matrices $A, B, C \in R^{n \times n}$, given any small positive number $\epsilon$ and appropriative positive number $\omega$ such that $0<$ $\omega<1$. Choose any initial matrix $X(0)$. Set $k:=1$.

Step 2. If $\delta_{k}=\left\|A X^{s s}(k)+X^{s s}(k) B-C\right\|_{F} /\|C\|_{F}<\epsilon$, stop; otherwise, go to Step 3.

Step 3. Update the sequences

$$
\begin{aligned}
& X_{1}(k)=X(k-1) \\
& -\mu_{1}\left[A^{T}(A X(k-1)+X(k-1) B-C)\right. \\
& \left.+(A X(k-1)+X(k-1) B-C) B^{T}\right], \\
& X_{2}(k)=X(k-1) \\
& \quad-\mu_{2}\left[B\left(B^{T} X(k-1)+X(k-1) A^{T}+C^{T}\right)\right. \\
& \left.\quad+\left(B^{T} X(k-1)+X(k-1) A^{T}+C^{T}\right) A\right], \\
& X(k)=\omega X_{1}(k)+(1-\omega) X_{2}(k), \\
& X^{s s}(k)=\frac{X(k)-X^{T}(k)}{2} .
\end{aligned}
$$

Step 4. Set $k:=k+1$; return to Step 2 .

Similarly, we have the following theorem to ensure the convergence of the Algorithm 10.

Theorem 11. Assume the matrix equations (30) have a unique solution $X^{*}$; then the iterative sequence $\{X(k)\}$ generated by the Algorithm 10 converges to $X^{*}$, if

$$
\begin{aligned}
& 0<\mu_{1}<\frac{1}{\omega\left(\|A\|_{2}^{2}+\|B\|_{2}^{2}\right)}, \\
& 0<\mu_{2}<\frac{1}{(1-\omega)\left(\|A\|_{2}^{2}+\|B\|_{2}^{2}\right)} ;
\end{aligned}
$$

that is, $\lim _{k \rightarrow \infty} X(k)=X^{*}$ or the error $X(k)-X^{*}$ converges to zero for any initial value $X(0)$.

Furthermore, the sequence $\left\{X^{s s}(k)\right\}$ converges to $X^{s s}$, where $X^{s s}$ is the unique skew symmetric solution of (1).

\section{Numerical Examples}

In this section, two numerical examples are used to show the efficiency of the RGI Method. All the computations were performed on Intel ${ }^{\circledR}$ Core $^{\mathrm{TM}}$ i7-4500U CPU @ $1.80 \mathrm{GHZ}$ 2.40 GHZ system by using MATLAB 7.0. EER is the Frobenius norm of absolute error matrices which is defined to be EER = $\left\|X(k)-X^{*}\right\|_{F}$, where $X(k)$ is the $k$ th iteration result for the RGI Method.

Example 1. In matrix equation (1), we choose

$$
\begin{aligned}
& A=\left(\begin{array}{cccc}
4.5 & 3.5 & 3.0 & -5.5 \\
-5.0 & -6.0 & -3.0 & 2.0 \\
4.0 & 2.0 & 5.0 & -3.0 \\
1.5 & 1.5 & 0.0 & -0.5
\end{array}\right), \\
& B=\left(\begin{array}{cccc}
-1.5 & -6.5 & -3.0 & -2.5 \\
1.0 & 6.0 & 3.0 & 1.0 \\
-4.0 & -5.0 & -4.0 & 0.0 \\
-1.5 & -1.5 & 0.0 & -0.5
\end{array}\right), \\
& C=\left(\begin{array}{cccc}
-5.5 & 12.5 & 11.0 & 4.5 \\
3.5 & -3.5 & 2.0 & -4.5 \\
-8.5 & -10.5 & -1.0 & -1.5 \\
2.0 & 0.0 & 0.0 & -1.0
\end{array}\right) .
\end{aligned}
$$

It is easy to show that the matrix equation (1) is consistent and has unique symmetric solution. By computing, the unique symmetric solution $X^{s}$ is given as

$$
X^{s}=\left(\begin{array}{cccc}
-1 & 1 & 0 & 1 \\
1 & 0 & -1 & 0 \\
0 & -1 & 1 & -1 \\
1 & 0 & -1 & 0
\end{array}\right)
$$

Taking $X(0)=10^{-6} \mathbf{1}_{2 \times 3}$ and applying the RGI Method (Algorithm 7) to compute the symmetric solution of $A X+$ $X B=C$, the sum of $\|A\|_{2}^{2}+\|B\|_{2}^{2}$ is 962.2175. When taking $\omega=0.4, \mu_{1}=0.0026$, and $\mu_{2}=0.0017$, the iterative errors $\delta_{k}$ versus $k$ are shown in Figure 1 , where $\delta_{k}=\left\|X^{s}(k)-X^{s}\right\|_{F}^{2}$. 


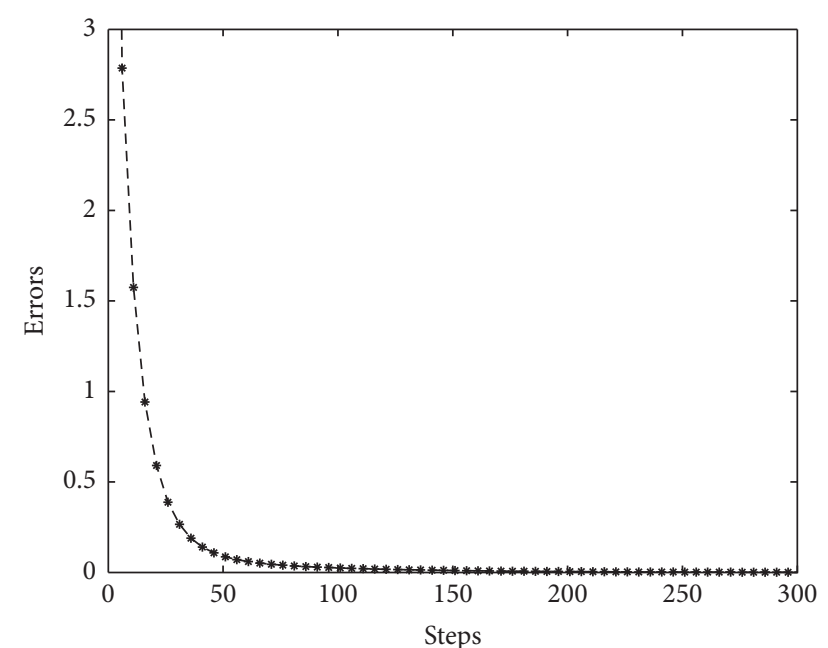

FIgURE 1: Convergence cure of symmetric solution $\left(\omega=0.4, \mu_{1}=\right.$ 0.0026 , and $\left.\mu_{2}=0.0017\right)$.

Example 2. In matrix equation (1), if we choose

$$
\begin{aligned}
A & =\left(\begin{array}{cccc}
4.5 & 8.5 & 3.0 & -5.5 \\
-5.0 & -9.0 & -3.0 & 7.0 \\
8.0 & 14.0 & 5.0 & -12.0 \\
1.5 & 1.5 & 0.0 & -0.5
\end{array}\right), \\
B & =\left(\begin{array}{cccc}
-1.5 & -6.5 & -3.0 & -2.5 \\
1.0 & 6.0 & 3.0 & 1.0 \\
-4.0 & -10.0 & -4.0 & 0.0 \\
-1.5 & -1.5 & 0.0 & -0.5
\end{array}\right), \\
C & =\left(\begin{array}{cccc}
-11.0 & 8.0 & 28.5 & 0.5 \\
7.5 & -2.5 & -27.0 & 6.5 \\
-19.5 & -19.5 & 34.0 & -3.5 \\
4.0 & 25.0 & 13.5 & 3.5
\end{array}\right),
\end{aligned}
$$

it is easy to check that the matrix equation (1) is consistent and has unique skew symmetric solution. The unique skew symmetric solution $X^{s s}$ is computed as

$$
X^{s s}=\left(\begin{array}{cccc}
0 & 2 & 0 & 1 \\
-2 & 0 & 2 & -1 \\
0 & -2 & 0 & 1 \\
-1 & 1 & -1 & 0
\end{array}\right) .
$$

Also taking $X(0)=10^{-6} \mathbf{1}_{2 \times 3}$ and $\omega=0.5, \mu_{1}=0.0021$, and $\mu_{2}=0.0021$, then using Algorithm 10, the convergence cure of skew symmetric solution is shown in Figure 2.

\section{Conflicts of Interest}

The authors declare that they have no conflicts of interest.

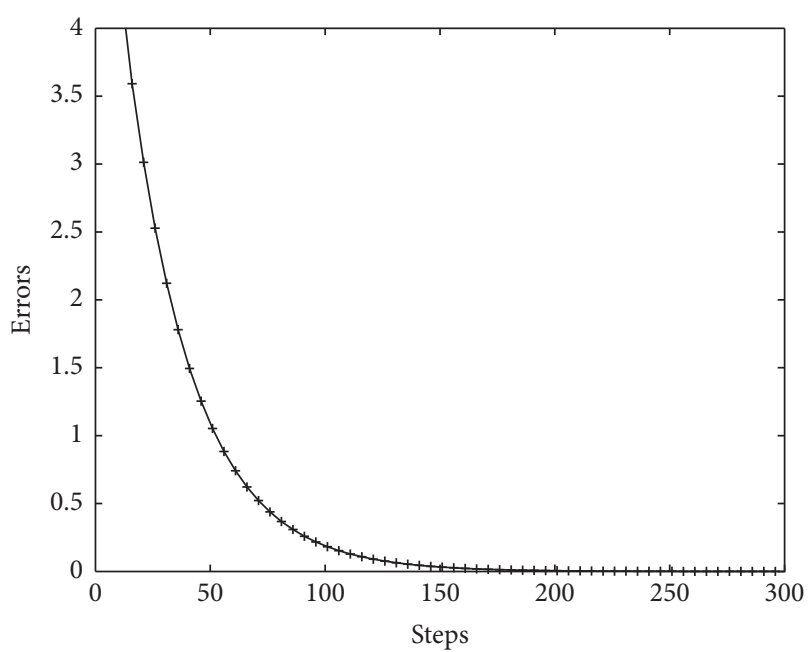

FIGURE 2: Convergence cure of skew symmetric solution $(\omega=0.5$, $\mu_{1}=0.0021$, and $\left.\mu_{2}=0.0021\right)$.

\section{Acknowledgments}

This project was supported by NSF China (no. 11471122), Anhui Provincial Natural Science Foundation (no. 1508085MA12), Key Projects of Anhui Provincial University Excellent Talent Support Program (no. gxyqZD2016188), and the University Natural Science Research Key Project of Anhui Province (no. KJ2015A161).

\section{References}

[1] S. P. Bhattacharyya and E. de Souza, "Pole assignment via Sylvester's equation," Systems \& Control Letters, vol. 1, no. 4, pp. 261-263, 1982.

[2] G. R. Duan, "Solutions to matrix equation $A V+B W=V F$ and their application to eigenstructure assignment in linear systems," IEEE Transactions on Automatic Control, vol. 38, no. 2, pp. 276-280, 1993.

[3] G. R. Duan, "On the solution to the Sylvester matrix equation $\mathrm{AV}+\mathrm{BW}=\mathrm{EVF}$, IEEE Transactions on Automatic Control, vol. 41, no. 4, pp. 612-614, 1996.

[4] B. Zhou and G. R. Duan, "A new solution to the generalized Sylvester matrix equation AV-EVF=BW," Systems \& Control Letters, vol. 55, no. 3, pp. 193-198, 2006.

[5] T. S. Hu, Z. L. Lin, and J. Lam, "Unified gradient approach to performance optimization under a pole assignment constraint," Journal of Optimization Theory and Applications, vol. 121, no. 2, pp. 361-383, 2004.

[6] J. Lam and W. Y. Yan, "A gradient flow approach to the robust pole-placement problem," International Journal of Robust and Nonlinear Control, vol. 5, no. 3, pp. 175-185, 1995.

[7] J. Lam and W.-Y. Yan, "Pole assignment with optimal spectral conditioning," Systems \& Control Letters, vol. 29, no. 5, pp. 241253, 1997.

[8] J. Lam, W.-Y. Yan, and T. Hu, "Pole assignment with eigenvalue and stability robustness," International Journal of Control, vol. 72, no. 13, pp. 1165-1174, 1999.

[9] B. N. Datta, W.-W. Lin, and J.-N. Wang, "Robust partial pole assignment for vibrating systems with aerodynamic effects," 
IEEE Transactions on Automatic Control, vol. 51, no. 12, pp. 1979-1984, 2006.

[10] B. Zhou and G. R. Duan, "Parametric approach for the normal Luenberger function observer design in second-order linear systems," in Proceedings of the 45th IEEE Conference on Decision and Control, pp. 1423-1428, San Diego, Calif, USA, 2006.

[11] D. L. Chu and P. Van Dooren, "A novel numerical method for exact model matching problem with stability," Automatica, vol. 42, no. 10, pp. 1697-1704, 2006.

[12] D. L. Chu, H. C. Chan, and D. W. C. Ho, "Regularization of singular systems by derivative and proportional output feedback," SIAM Journal on Matrix Analysis and Applications, vol. 19, no. 1, pp. 21-38, 1998.

[13] D. L. Chu, V. Mehrmann, and N. K. Nichols, "Minimum norm regularization of descriptor systems by mixed output feedback," Linear Algebra and Its Applications, vol. 296, no. 1-3, pp. 39-77, 1999.

[14] D. L. Chu and V. Mehrmann, "Disturbance decoupling for descriptor systems by state feedback," SIAM Journal on Control and Optimization, vol. 38, no. 6, pp. 1830-1858, 2000.

[15] D. L. Chu and R. C. E. Tan, "Numerically reliable computing for the row by row decoupling problem with stability," SIAM Journal on Matrix Analysis and Applications, vol. 23, no. 4, pp. 1143-1170, 2002.

[16] G. H. Golub, S. Nash, and C. Van Loan, "A Hessenberg-Schur method for the problem $A X-X B=C$," IEEE Transactions on Automatics Control, vol. 24, pp. 909-913, 1979.

[17] R. H. Bartels and G. W. Stewart, "Algorithm 432: Solution of the matrix equation $A X-X B=C$," Communications of the ACM, vol. 15, no. 9, pp. 820-826, 1972.

[18] Y.-B. Deng, Z.-Z. Bai, and Y.-H. Gao, "Iterative orthogonal direction methods for Hermitian minimum norm solutions of two consistent matrix equations," Numerical Linear Algebra with Applications, vol. 13, no. 10, pp. 801-823, 2006.

[19] Z.-Z. Bai, "On Hermitian and skew-Hermitian splitting iteration methods for continuous Sylvester equations," Journal of Computational Mathematics, vol. 29, no. 2, pp. 185-198, 2011.

[20] A. Bouhamidi and K. Jbilou, "A note on the numerical approximate solutions for generalized Sylvester matrix equations with applications," Applied Mathematics and Computation, vol. 206, no. 2, pp. 687-694, 2008.

[21] A. Kaabi, F. Toutounian, and A. Kerayechian, "Preconditioned Galerkin and minimal residual methods for solving Sylvester equations," Applied Mathematics and Computation, vol. 181, no. 2, pp. 1208-1214, 2006.

[22] A. Kaabi, A. Kerayechian, and F. Toutounian, "A new version of successive approximations method for solving Sylvester matrix equations," Applied Mathematics and Computation, vol. 186, no. 1, pp. 638-645, 2007.

[23] Y.-Q. Lin, "Implicitly restarted global FOM and GMRES for nonsymmetric matrix equations and Sylvester equations," Applied Mathematics and Computation, vol. 167, no. 2, pp. 10041025, 2005.

[24] Y. Lin, "Minimal residual methods augmented with eigenvectors for solving SYLvester equations and generalized SYLvester equations," Applied Mathematics and Computation, vol. 181, no. 1, pp. 487-499, 2006.

[25] D. Khojasteh Salkuyeh and F. Toutounian, "New approaches for solving large Sylvester equations," Applied Mathematics and Computation, vol. 173, no. 1, pp. 9-18, 2006.
[26] J.-J. Zhang, "A note on the iterative solutions of general coupled matrix equation," Applied Mathematics and Computation, vol. 217, no. 22, pp. 9380-9386, 2011.

[27] F. Ding and T. Chen, "Hierarchical gradient-based identification of multivariable discrete-time systems," Automatica, vol. 41, no. 2, pp. 315-325, 2005.

[28] F. Ding and T. Chen, "Gradient-based iterative algorithms for solving a class of matrix equations," IEEE Transactions on Automatic Control, vol. 50, no. 8, pp. 1216-1221, 2005.

[29] F. Ding and T. Chen, "Hierarchical identification of lifted statespace models for general dual-rate systems," IEEE Transactions on Circuits and Systems. I. Regular Papers, vol. 52, no. 6, pp. 11791187, 2005.

[30] J.-P. Chehab and M. Raydan, "An implicit preconditioning strategy for large-scale generalized Sylvester equations," Applied Mathematics and Computation, vol. 217, no. 21, pp. 8793-8803, 2011.

[31] F. Ding and T. Chen, "On iterative solutions of general coupled matrix equations," SIAM Journal on Control and Optimization, vol. 44, no. 6, pp. 2269-2284, 2006.

[32] F. Ding, P. X. Liu, and J. Ding, "Iterative solutions of the generalized Sylvester matrix equations by using the hierarchical identification principle," Applied Mathematics and Computation, vol. 197, no. 1, pp. 41-50, 2008.

[33] A.-G. Wu, X. Zeng, G.-R. Duan, and W.-J. Wu, "Iterative solutions to the extended Sylvester-conjugate matrix equations," Applied Mathematics and Computation, vol. 217, no. 1, pp. 130142, 2010.

[34] Q. Niu, X. Wang, and L.-Z. Lu, "A relaxed gradient based algorithm for solving Sylvester equations," Asian Journal of Control, vol. 13, no. 3, pp. 461-464, 2011.

[35] X. Wang, L. Dai, and D. Liao, "A modified gradient based algorithm for solving Sylvester equations," Applied Mathematics and Computation, vol. 218, no. 9, pp. 5620-5628, 2012.

[36] Y.-J. Xie and C.-F. Ma, “The accelerated gradient based iterative algorithm for solving a class of generalized Sylvester-transpose matrix equation," Applied Mathematics and Computation, vol. 273, pp. 1257-1269, 2016.

[37] Y. Xie and C. Ma, "Iterative methods to solve the generalized coupled Sylvester-conjugate matrix equations for obtaining the centrally symmetric (centrally antisymmetric) matrix solutions," Journal of Applied Mathematics, vol. 2014, Article ID 515816, 17 pages, 2014.

[38] Y. Xie, N. Huang, and C. Ma, "Iterative method to solve the generalized coupled Sylvester-transpose linear matrix equations over reflexive or anti-reflexive matrix," Computers \& Mathematics with Applications, vol. 67, no. 11, pp. 2071-2084, 2014. 


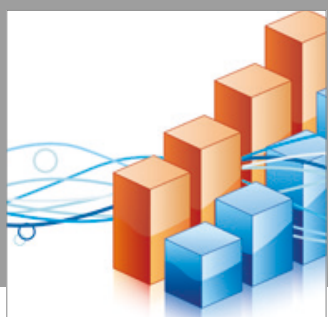

Advances in

Operations Research

vatersals

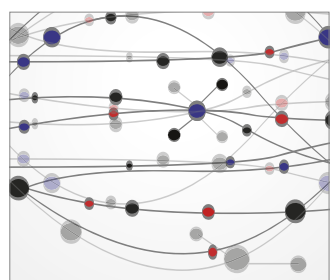

\section{The Scientific} World Journal
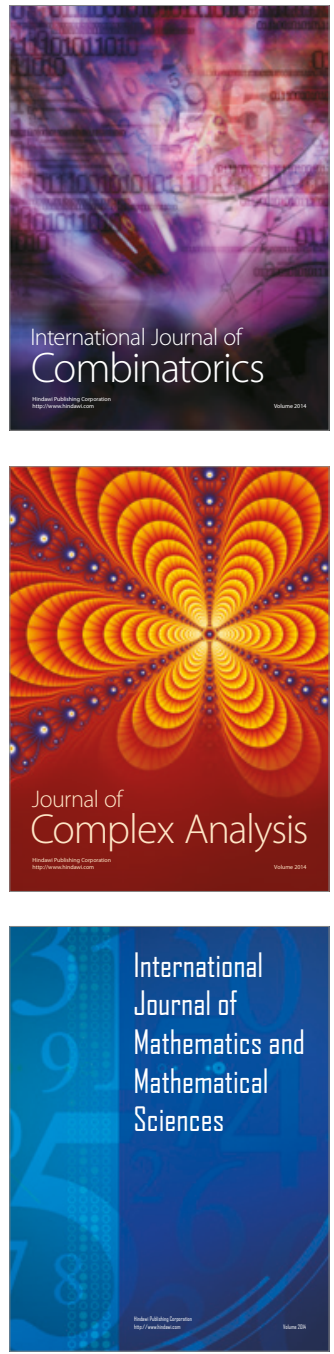
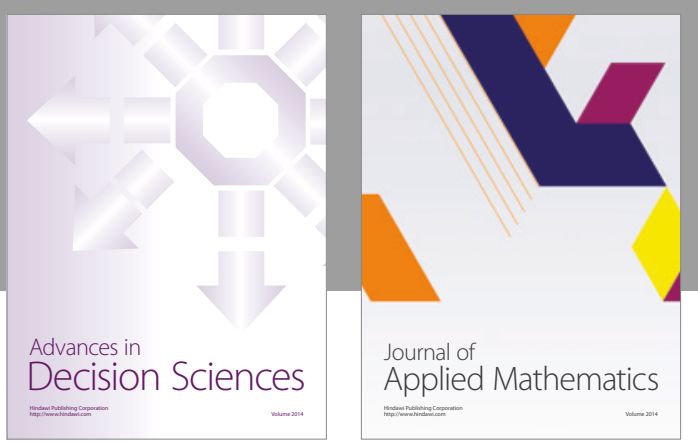

Algebra

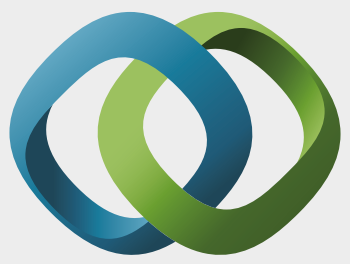

\section{Hindawi}

Submit your manuscripts at

https://www.hindawi.com
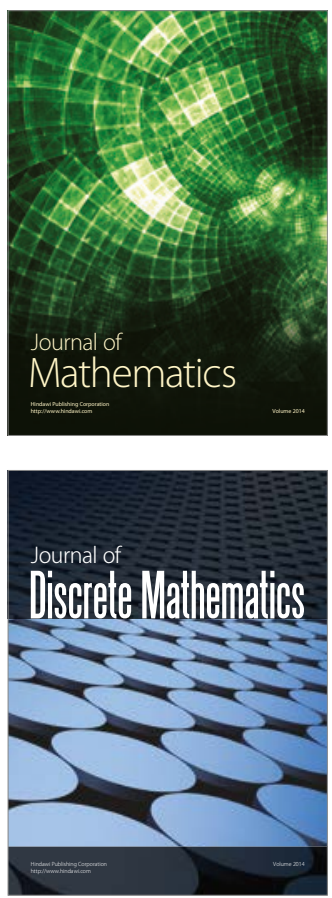

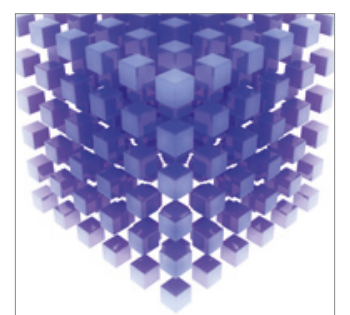

Mathematical Problems in Engineering
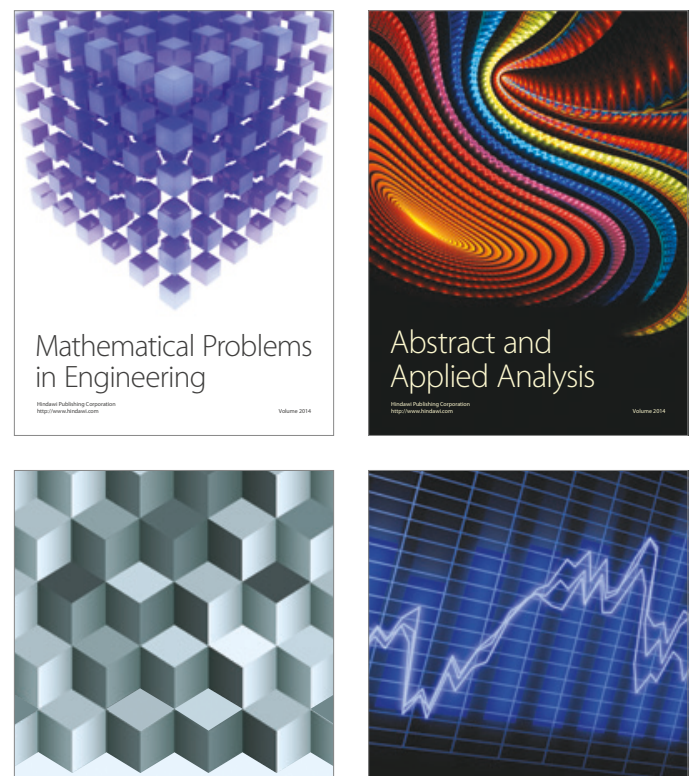

Journal of

Function Spaces

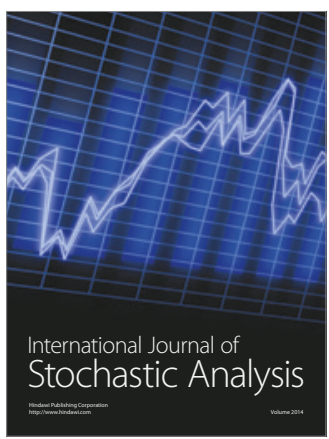

Probability and Statistics
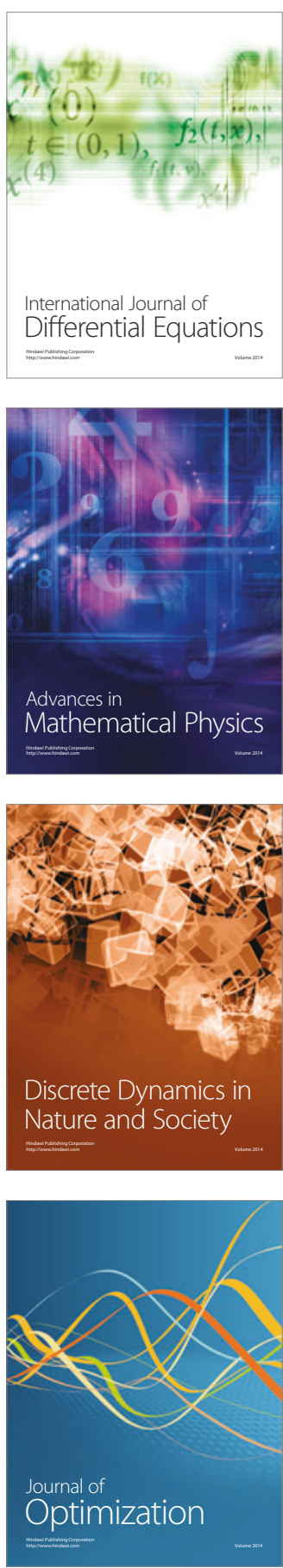